\title{
Enhancing the Implementation of a First-order Equivalent Thermal Parameter Model to Enable Accurate and Robust Building Thermal Response Prediction
}

\author{
Kathryn Hinkelman ${ }^{1}$, Sen Huang ${ }^{2}$, Jing Wang ${ }^{1}$, Jianming Lian², Wangda Zuo ${ }^{1}$ \\ ${ }^{1}$ University of Colorado, Boulder, CO, USA \\ ${ }^{2}$ Pacific Northwest National Laboratory, Richland, WA, USA
}

\begin{abstract}
This paper proposes a novel method to implement a first-order equivalent thermal parameter (ETP) model, a commonly used model to provide building thermal response prediction for advanced building controls. This method reformulates the existing implementation method to allow the ETP model to capture varying heat disturbance and non-linearity in the zone temperature evolution. It also guarantees that the prediction from the ETP model is always reasonable by considering more physical constraints. To evaluate performance, we implemented an ETP model with the proposed method for a medium-office building and predicted the zone temperatures for one day. Prediction results are compared against those from an ETP model implemented through a common approach and a lookup-table model. Results suggest that the ETP model implemented by the proposed method can yield more accurate results compared to the other two models; additionally, the ETP model implemented by the proposed method demonstrates similar capabilities in capturing non-linear behaviors as the lookup-table model.
\end{abstract}

\section{Introduction}

In the United States, buildings consume $74 \%$ of the total electricity production (Energy Information Administration, 2017). High building energy consumption not only leads to high operational costs but also has substantial environmental impacts (Omer, 2009). While building energy consumption remains an important concern, over the past decade, buildings have also been recognized as potentially valuable resources for realizing a reliable and robust power grid (Dodrill, 2011). To these ends, advanced controls have demonstrated success in enhancing the energy efficiency of building operations and its capability to provide grid services (Fernandez et al., 2017; Huang et al., 2016; Hao et al., 2017a).

When utilizing advance controls, however, it is important to avoid any unacceptable sacrifices on the services buildings provide, including maintaining a comfortable indoor thermal environment. As a re- sult, it is often necessary to predict the thermal behavior of buildings, indicated by indoor temperature, in response to control actions and other disturbances, such as weather conditions or occupant behaviors. In fact, zone temperature prediction has been gaining attentions ever since the nineties, and many prediction models have been proposed (Inard et al., 1996; Crawley et al., 2001; Ruano et al., 2006; Taylor et al., 2008; Alasha'ary et al., 2009; Lu and Viljanen, 2009; Mustafaraj et al., 2011; Lin et al., 2012; Huang et al., 2013; Chinde et al., 2015). Those models can be categorized into three categories: white-box models, black-box models, and gray-box models.

White-box models evaluate the temperature evolution based on the first-principle laws, such as heat transfer and air flow dynamics, and knowledge of studied buildings, such as the thermal properties of the envelope. Provided enough detailed knowledge, they can yield relatively good prediction accurate but tend to be computationally intensive (Crawley et al., 2001).

Black-box models learn the mapping between zone temperature and the operating condition purely from historical data, with little requirement on knowledge of studied buildings. Their accuracy may be subject to how well the historical data covers all the possible operating conditions, and thus their extrapolation capability is questionable. However, their computational demand is usually low since usually no sophisticated mathematical operations are needed.

Gray-box models first establish the prediction mechanisms with unknown coefficients, based on high-level information of studied buildings, such as whether the studied zone is a perimeter zone or an internal zone. They then estimate the values of the unknown coefficients by performing regression on the historical data. Compared to the white-box methods, the graybox methods require less information regarding the building information, which may be expensive to obtain; while compared to the black-box methods, the gray-box methods can have better performance in the extrapolation (Chinde et al., 2015), and thus they depend less on the quality of the historical data. 
Due to their advantages over other models, the graybox models are commonly used when developing advanced building controls (Lin et al., 2015; Hao et al., 2017b, 2018; Vivian et al., 2017). Frequently, researchers tend to use linear equations when implementing the gray-box models to further simplify the deployment and minimize computational demand. Despite their promising nature, existing methods to implement gray-box models contain three primary limitations that need to be overcome in order to enable large-scale applications:

1. They often have difficulties capturing non-linear behaviors.

2. They may not be able to consider varying disturbances.

3. They may generate unreasonable values for unknown coefficient during regression.

With the first limitation, there is an obvious difficulty capturing non-linear behaviors with linear equations, and non-linearity is notably common in buildings. For example, the change of the set point may not immediately affect the zone temperature because of the thermal or mechanical inertia. With the second limitation, disturbances that are present for zone temperature predictions may not be a function of the zone temperature. Often defined as the heat gain, disturbances are usually considered as one constant term when implementing the gray-box model. However, disturbances may vary by time. For example, the solar irradiation is one major heat gain and can vary dramatically within one day. Having one constant term to represent disturbances may lead to significant prediction error. Lastly with the third limitation, as mentioned above, gray-box methods need to estimate the values of the unknown coefficients by performing regression analysis. Least square estimation (LSE) (Whittle, 1963) is a popular regression approach; however, the obtained values do not necessarily have correct physical meanings. For example, it is possible that the regression results may indicate the temperature will rise when increasing the amount of cooling energy.

To address these limitations, we propose a novel method to implement the first-order equivalent thermal parameter (ETP) model, which is a commonly used gray-box model. Compared to existing methods, the proposed method can capture non-linear behaviors, better represent the varying disturbances, and guarantee that the prediction are meaningful. To evaluate the performance, we applied the proposed method in predicting the zone temperature for a medium-office building, over the course of one day. Predictions are compared against two existing methods: the gray-box model implemented with the LSE approach and a look-up table, a common black-box model.

The remaining sections of this paper are organized as follows. First, we introduce the ETP model and current implementations. Then, we describe our proposed method to implement the first-order ETP model. With the methodology detailed, its performance is evaluated through a case study and the results are discussed. Finally, we present conclusions and future work.

\section{First-order ETP Model}

ETP models represent the thermodynamics of a building through an electrical circuit analogy with a lumped thermal resistance $R$ and capacitance $C$. A first-order ETP model is based on the First Law of Thermodynamics described as:

$$
C \frac{d T}{d t}=\frac{T_{o}-T}{R}+\dot{Q}_{h v a c}+w
$$

where $T$ denotes the zone temperature; $t$ denotes the time; $T_{o}$ denotes the outdoor air temperature; $\dot{Q}_{h v a c}$ denotes the cooling/heating energy from the heating, ventilation, and air conditioning (HVAC) system; and $w$ denotes the disturbance, such as the solar irradiation. Equation (1) contains three inputs $\left(T_{o}, \dot{Q}_{\text {hvac }}\right.$, and $w)$ and two parameters $(C$ and $R)$.

\section{Existing Implementation}

Equation (1) is a derivative equation and may cause difficulties in real-world deployment. To simplify the deployment process, a commonly used implementation method is described as follows. First, $w$ is assumed to be constant, and a discrete form for equation (1) is developed:

$$
C \frac{T^{k+1}-T^{k}}{\delta t}=\frac{T_{o}^{k}-T^{k}}{R}+\dot{Q}_{h v a c}^{k}+c_{0}
$$

where $k$ denotes the time step of the prediction horizon; $\delta t$ denotes the discrete time interval; and $c_{0}$ denotes the constant disturbance.

Then, for the HVAC system that is managed by on-off control, the cooling/heating energy from the HVAC system is assumed to be constant when the system is on. In this case, equation (2) becomes:

$$
C \frac{T^{k+1}-T^{k}}{\delta t}=\frac{T_{o}^{k}-T^{k}}{R}+s^{k} \dot{Q}_{h v a c, r}+c_{0}
$$

where $s^{k}$ denotes the on-off status of the HVAC system ( 0 for off and 1 for on) at the $k$ th time step. $\dot{Q}_{h v a c, r}$ denotes the rated cooling/heating energy provided by the HVAC system.

Finally, equation (3) is reformulated as:

$$
\left\{\begin{array}{l}
T^{k+1}=c_{1} T^{k}+c_{2} T_{o}^{k}+c_{3} s^{k} \dot{Q}_{h v a c, r}+c_{0} \\
c_{1}=1-\frac{\delta t}{R C} \\
c_{2}=\frac{\delta t}{R C} \\
c_{3}=\frac{\delta t}{C}
\end{array}\right.
$$

In equation (4), there are three inputs $\left(T^{k}, T_{o}\right.$, and $\left.s^{k}\right)$ and four parameters $\left(c_{0}, c_{1}, c_{2}, c_{3}\right)$. The values of 
the parameters are obtained via regression. As previously discussed, a commonly used regression method is a least square regression analysis (or LSE), which solves the following unconstrained optimization problem:

$$
\min _{c_{0}, c_{1}, c_{2}, c_{3}} \sum_{i=1}^{N}\left(T^{k}-\hat{T}^{k}\right)^{2}
$$

where $\hat{T}^{k}$ is the actual zone temperature sampled at the $k$ th time step, and $N$ is the number of the data points considered in the regression.

\section{Proposed Implementation}

As mentioned above, the existing method to implement the ETP model has three major limitations: (1) They often have difficulties capturing non-linear behaviors; (2) they may not be able to consider varying disturbances; and (3) they may generate unreasonable values for unknown coefficients during regression.

To address the first limitation, we changed equation (3) into:

$$
C \frac{T^{k+1}-T^{k}}{\delta t}=\frac{T_{o}^{k}-T^{k}}{R}+s^{k-n} \dot{Q}_{h v a c, r}+c_{0}
$$

where $n$ is a parameter to reflect the delay between the time when a control action is made and the time when the studied zone begins to respond to the control action.

To address the second limitation, we further modified equation (6) by assuming that disturbances remain constant during a short period from the $k_{i-1}$ th time step to the $k_{i}$ th time step:

$$
\left\{\begin{array}{l}
C^{1} \frac{T^{k+1}-T^{k}}{\delta t}=\frac{T_{o}^{k}-T^{k}}{R^{1}}+s^{k-n} \dot{Q}_{h v a c, r}+c_{0}^{1} \\
\left(0<k \leq k_{1}\right) \\
\quad \vdots \\
C^{i} \frac{T^{k+1}-T^{k}}{\delta t}=\frac{T_{o}^{k}-T^{k}}{R^{i}}+s^{k-n} \dot{Q}_{h v a c, r}+c_{0}^{i} \\
\left(k_{i-1}<k \leq k_{i}\right) \\
\quad \vdots \\
C^{m} \frac{T^{k+1}-T^{k}}{\delta t}=\frac{T_{o}^{k}-T^{k}}{R^{m}}+s^{k-n} \dot{Q}_{h v a c, r}+c_{0}^{m} \\
\left(k_{i-1}<k<k_{m}\right)
\end{array}\right.
$$

where $i$ is the index for the sub-period with constant disturbances $(i=1,2,3 \ldots m)$, and $m$ is the total number of the sub-periods in the prediction horizon. For example, a single-day prediction horizon can be divided into a total of $m=24$ sub-periods, and the index $i=1,2,3 \ldots 24$ represents each hour in the day. Lastly, $C^{i}, R^{i}$, and $c_{0}^{i}$ are, respectively, the thermal capacitance, the thermal resistance, and the disturbances at the $i$ th sub-period.

There are many ways to determine the values for $k_{i-1}$ and $k_{i}$. The simplest method is to assume the subperiods are evenly divided, such as,

$$
k_{i}=i \frac{N}{m}
$$

where $N$ is the total number of intervals in the prediction horizon.

To address the third limitation, we conducted the following regression. First, for each sub-period, we reformulated equation (7) as:

$$
\left\{\begin{array}{l}
T^{k+1}=c_{1}^{i} T^{k}+c_{2}^{i} T_{o}^{k}+c_{3}^{i} s^{k-n} \dot{Q}_{h v a c, r}+c_{0}^{i} \\
c_{1}^{i}=1-\frac{\delta t}{R_{i} C_{i}} \\
c_{2}^{i}=\frac{\delta t}{R_{i} C_{i}} \\
c_{3}^{i}=\frac{\delta t}{C_{i}}
\end{array}\right.
$$

We then assumed $n=0$ and solved the following optimization problem:

$$
\begin{aligned}
\min _{c_{0}^{i}, c_{1}^{i}, c_{2}^{i}, c_{3}^{i}} & \sum_{s=1}^{N^{i}}\left(T^{k}-\hat{T}^{k}\right)^{2} \\
\text { s.t. } & c_{0}^{i}>0 \\
& c_{1}^{i}>0 \\
& c_{2}^{i}>0 \\
& c_{3}^{i}>0 \\
& c_{1}^{i}+c_{2}^{i}=1
\end{aligned}
$$

where $N^{i}$ is the number of data points that lie in the sub-period $\left[k_{i-1}, k_{i}\right]$.

Second, after obtaining the values for $c_{0}^{i}, c_{1}^{i}, c_{2}^{i}$, and $c_{3}^{i}$, we solve the following optimization problem:

$$
\begin{array}{ll}
\min _{n} & \sum_{i=1}^{N}\left(T^{k}-\hat{T}^{k}\right)^{2} \\
\text { s.t. } & n=1,2,3, \ldots
\end{array}
$$

By considering more constraints when conducting the regression, we can guarantee the generated coefficients are consistent with the laws of physics. The above optimization problems are implemented and solved with a Python package called scipy.optimize (Jones et al., 2001).

In summation, the proposed methodology addresses all three limitations of existing ETP implementation methods by reformulating the discrete function and revising the method to perform the regression. We expect the proposed method can increase the prediction accuracy and eliminate the possibility of generating unreasonable results. In the following section, we evaluate the performance of the proposed method against existing methods via a case study.

\section{Case Study}

In this case study, we considered a real building located in eastern Washington, U.S.. The test building is served by 10 single-zone, constant air volume rooftop units (RTU). However, we only considered one RTU and the associated thermal zone in this study. This section first introduces the thermal zone. 
The training and testing data are then discussed, followed by the prediction settings. Finally, we present the evaluation results and associated analysis.

\section{Thermal Zone}

The studied thermal zone, as shown in Figure 1, is located in the north-west side of the building. It contains four private office rooms (total area: $55.2 \mathrm{~m}^{2}$ ) and has one north external wall and four west external walls. We selected this thermal zone because it is isolated from other areas of the building and thus has relatively less impact from other zones.

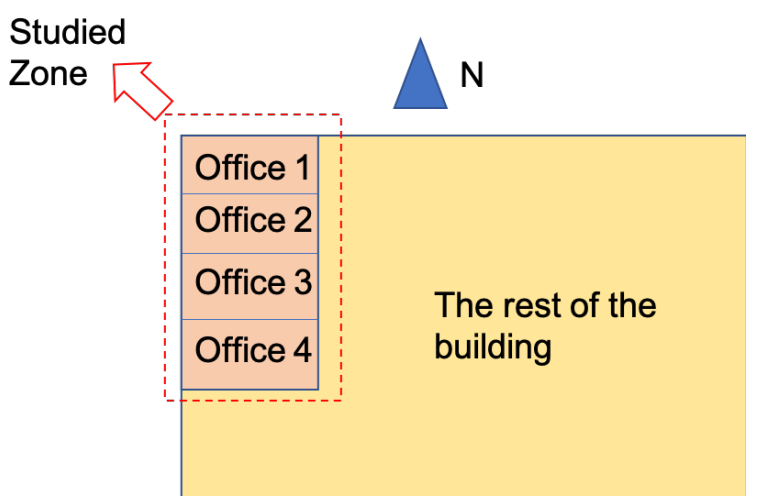

Figure 1: Building thermal zone schematic.

The RTU that serves this thermal zone has a rated cooling capacity of $7 \mathrm{~kW}$ and is controlled to maintain the average temperature of the four office rooms in a desired range. When only considering the cooling mode, the RTU has two modes of operation: (1) occupied and (2) unoccupied (Figure 2). During the occupied period, the supply fan is operating at a constant speed while the compressor is cycling based on the average temperature. The compressor is turned on only when the average temperature is larger than the cooling set point plus the dead band. Further, the compressor is not in the lock-off status (the compressor has a minimum off time). The compressor will be turned off when the average temperature is lower than the cooling set point minus the dead band. During the unoccupied period, the fan and the compressor are both cycling based on the average temperature, as can be seen in Figure 2 .

\section{Training and Testing Data}

Training and testing data are sampled every minute by the building management system. This data includes the compressor status (on/off), the outdoor air temperature, the average air temperature, and the sampled time. The data covers the period of one month (June, 2017), and only weekdays were considered in this study. As illustrated in Figure 3, we selected the data for June 7 th as the testing data set and the rest of the data as the training data set.

\section{Prediction Settings}

As mentioned in the previous section, the testing data set covers one whole day. For the proposed methodology, we selected a prediction interval of 1 minute
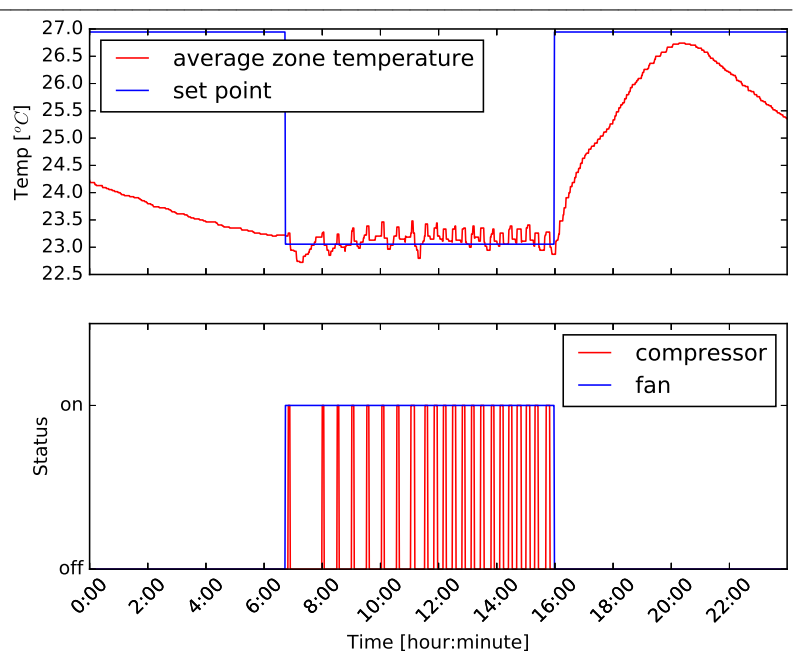

Figure 2: Typical RTU operation.

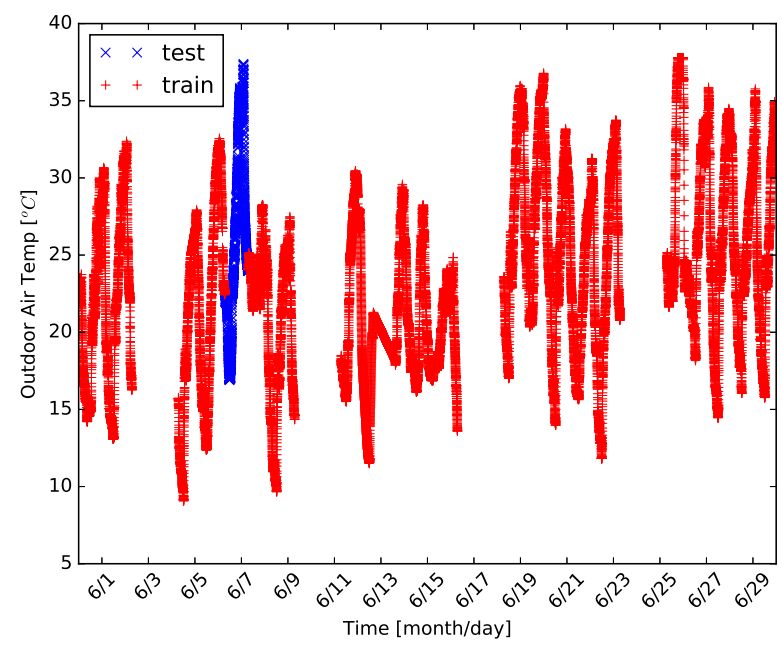

Figure 3: The training data and testing data.

and the total number of sub-periods $m=24$. With this, the total number of sub-intervals in the prediction horizon $N=1,440$, and each hour is treated as one sub-period.

To evaluate the performance of the proposed methodology, we compared prediction results with two existing approaches. First, the existing LSE method was used to implement the same ETP model as our proposed methodology. The procedure for LSE is detailed in section First-order ETP Model. For a second comparison, we also considered a look-up table method, a commonly used black-box approach.

The basic idea for the look-up table method is as follows. First, it divide the continuous space of the inputs into discrete segments. Then, the discrete segments are converted into a multiple-dimensional table that maps the inputs and the outputs based on the training data set. Since the look-up table doesn't need to assume that there are linear correlations between inputs and outputs, it is expected to better represent the non-linear features. Readers can find more information regarding the look-up table method in Huang et al. (2018). In this case, there are four inputs in the look-up table: outdoor temperature, zone temperature at the previous time step, the hour in- 
dex, and the compressor status. Since the hour index and the compressor status are already discrete, only the outdoor temperature and the zone temperature at the previous time step need to be discretized. The discretized interval we considered is $1.11^{\circ} \mathrm{C}\left(2^{\circ} \mathrm{F}\right)$.

For predicting the zone temperature with all three methods, we need three inputs: the outdoor temperature, the compressor status, and the zone temperature at the previous time step. To simplify the evaluation, we assume the outdoor temperature and the compressor status are obtained through ideal forecasts; thus, we used the measured values as the inputs for the zone temperature prediction rather than predicted outdoor temperatures and control actions. For the zone temperature at the previous time step, the real measurement for the first time step was used, while for the subsequent time steps, the prediction result at the previous time step was used as the input for the current time step.

\section{Results \& Discussion}

The prediction results indicate that no method provides a perfect prediction, as can be seen in Figure 4. The look-up table method generates the worst prediction; from 6:00 to 16:00, its prediction is constant. This is because the look-up table can't find the relevant information in the training data set; thus it just maintains the prediction from the last time step by default. This validates the statement that the blackbox models rely more on the richness of the training data set and work poorly when extrapolating the training data set. The LSE method generates reasonable predictions during unoccupied periods but worse predictions during the occupied period. We believe this is due to its inability to capture the varying disturbances, since disturbances are expected to change more dramatically during the occupied period. The proposed method generates the best prediction among the three methods, especially during the occupied period. The proposed method produces similar predictions during unoccupied period to the LSE method, but much accurate ones during the occupied period. This indicates that the proposed method provides a better way to handle the varying disturbances.

Figure 5 shows the detailed results regarding prediction errors. We can see that the proposed method generates slightly lower prediction error than the LSE method for the full day prediction; over the full day, the root mean square errors (RMSE) for the lookup table, the LSE method, and the proposed method are $0.631^{\circ} C, 0.489^{\circ} C$, and $0.263^{\circ} C$, respectively. The prediction errors for the proposed method are within the range from $-0.75^{\circ} \mathrm{C}$ to $0.75^{\circ} \mathrm{C}$, while those for the LSE method are within the range from $-1^{\circ} \mathrm{C}$ to $1^{\circ} \mathrm{C}$. The prediction errors for the look-up table method are within the range from $-2^{\circ} \mathrm{C}$ to $0.8^{\circ} \mathrm{C}$. If we only consider the occupied period, the prediction of the proposed method is much better than the other
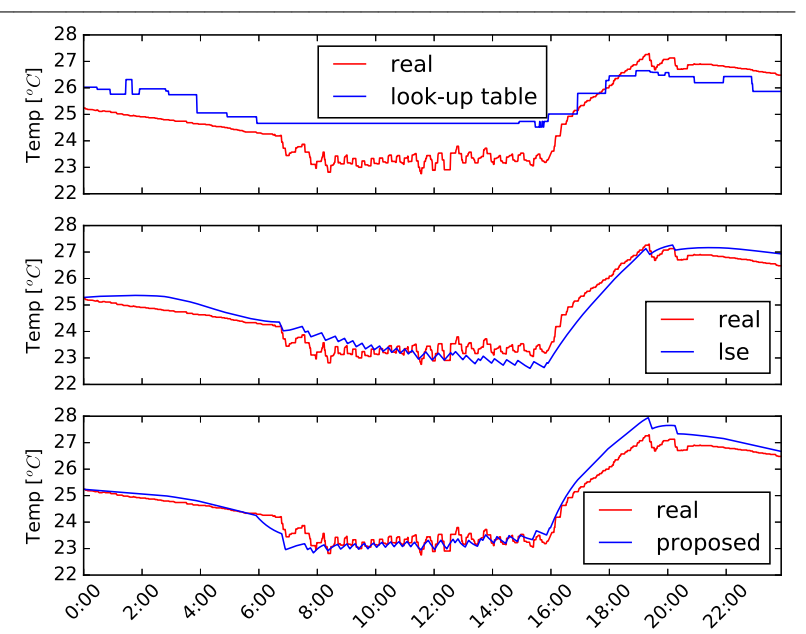

Figure 4: The full day prediction result.

two methods. The prediction errors for the proposed method are within the range from $-0.4^{\circ} \mathrm{C}$ to $0.5^{\circ} \mathrm{C}$. Those from the LSE method and the look-up table method are within the range from $-1^{\circ} \mathrm{C}$ to $0.8^{\circ} \mathrm{C}$, and from $-2^{\circ} C$ to $-1^{\circ} C$, respectively. Since the prediction on the occupied period is more important for implementing advanced building control, we believe the proposed method generates much better predictions.

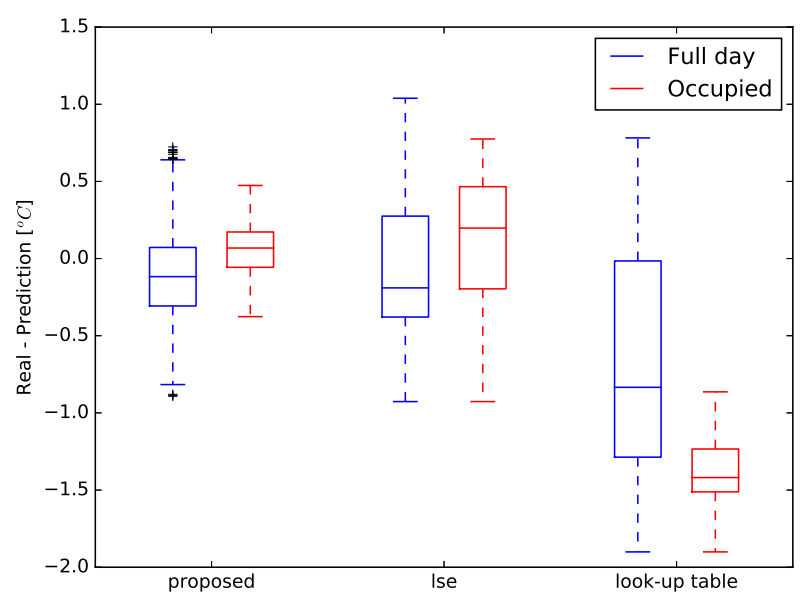

Figure 5: The full day prediction errors.

As mentioned above, the look-up table method stalls around 6:00 when the operation condition is not covered by the training data set. To understand how its performance changes when the prediction conditions are fully covered by the training data set, we reduced the prediction horizon from one day to a short 6 -hour period from 8:00 to 14:00. Figure 6 demonstrates the prediction results for this shorter prediction horizon. We can see the look-up table method produces a much better prediction compared to the full day prediction. This improvement emphasizes the importance of the training data set for the look-up table method. The proposed method produces similar prediction results to the look-up table method, and the prediction from the LSE method becomes the least accurate.

In addition, it is worth mentioning there exists notable delays between the LSE predictions and the 
real temperature values. For example, at around 11:15, the real zone temperature begins to drop while the LSE predicted temperature continues to increase. This indicates that the LSE method fails to capture the non-linearity that exists in the real data. On the contrary however, we don't observe similar delays in both the look-up table method and the proposed method. This result suggests that the proposed method has similar capability in capturing non-linear behaviors as the look-up table method.

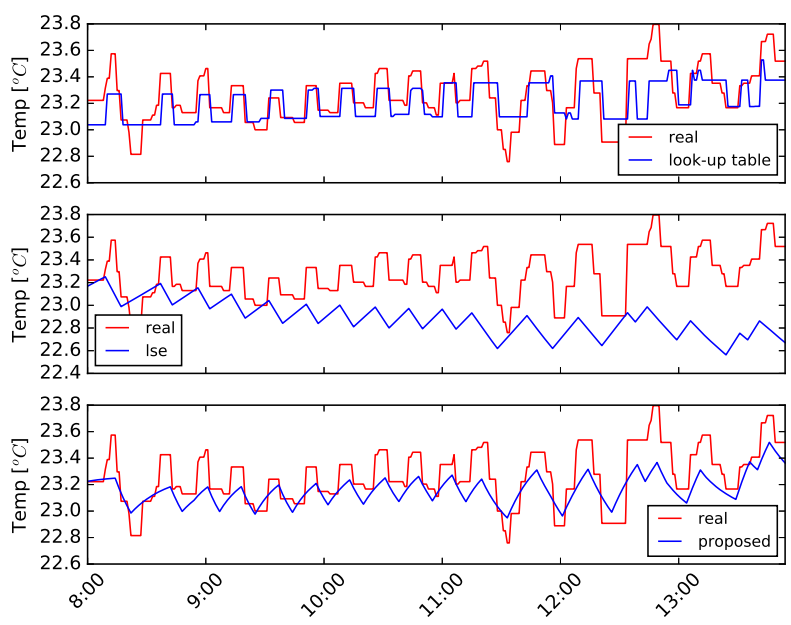

Figure 6: The prediction result with a short prediction horizon (8:00 to 14:00).

\section{Conclusion}

In this paper, we discussed the importance of zone temperature prediction for the development of advanced building controls and analyzed the advantages and disadvantages of the exiting methods. Based on the analysis, we proposed a new method to overcome the limitations that existing methods face when implementing a first-order ETP model, which is a commonly used gray-box model. To validate whether the proposed method can address the limitations, we performed a case study with real data from a medium office building in eastern Washington, U.S.

The results indicated that the proposed method can yield significantly better results compared to the LSE method and the look-up table method. We believe that the proposed method's enhanced prediction performance is largely due to its ability to better address the varying disturbances. For the look-up table method, its prediction accuracy highly depends on the richness of the training data set. In the case we considered, since the prediction of the previous time step will be used as the inputs for the prediction at the current time step, it is natural that the look-up table gets stuck at some points. This tendency causes difficulties when deploying look-up table control schemes. Therefore, we believe the proposed method can generally reduce the efforts to deploy the zone temperature prediction while still providing reasonably good predictions.
Furthermore, the proposed method demonstrated a similar capability in capturing non-linearity as the look-up table method. One of the major concerns regarding the linear regression model is that they tend to ignore the nonlinear nature of the studied system. However, by considering a constant time delay, the proposed method generates a similar prediction in terms of non-linearity compared to the look-up table method, which typically represents non-linear systems better than the first-order models.

While this study introduced new constraints to the optimization problem, we did not investigate how the proposed method generates better values for the regression coefficients by considering additional constraints. This is because we believe that this feature can be better assessed when the zone temperature prediction is integrated with advanced controls. In that case, we have more chances to use the prediction in the extrapolation for the what-if scenario analysis to generate an appropriate control action. As a result, these considerations will be included in a future study.

In addition, this study assumed the forecasts of the outdoor temperature and the control action are ideal. Thus, we used the measured values as the inputs for the zone temperature prediction. However, in the real-world applications, this assumption is unlikely valid. To provide a more realistic assessment, future studies will also include the prediction of the outdoor temperature and the control action in the evaluation. Furthermore, future investigations will expand upon this initial case study by testing the model on additional buildings, HVAC system designs, and in dedicated control applications.

\section{Acknowledgement}

This research was supported by the National Science Foundation under Award No. IIS-1802017: BIGDATA: Collaborative Research: IA: Big Data Analytics for Optimized Planning of Smart, Sustainable, and Connected Communities. This work has also been supported by the Fuel Cell Technologies Office of the U.S. Department of Energy's Office of Energy Efficiency and Renewable Energy. Lastly, this work emerged from the IBPSA Project 1, an internationally collaborative project conducted under the umbrella of the International Building Performance Simulation Association (IBPSA). Project 1 aims to develop and demonstrate a BIM/GIS and Modelica Framework for building and community energy system design and operation.

\section{References}

Alasha'ary, H., B. Moghtaderi, A. Page, and H. Sugo (2009). A neuro-fuzzy model for prediction of the indoor temperature in typical australian residential buildings. Energy and Buildings 41(7), 703-710. 
Chinde, V., J. C. Heylmun, A. Kohl, Z. Jiang, S. Sarkar, and A. Kelkar (2015). Comparative evaluation of control-oriented zone temperature prediction modeling strategies in buildings. In $A S M E$ 2015 Dynamic Systems and Control Conference, pp. V002T34A009-V002T34A009. American Society of Mechanical Engineers.

Crawley, D. B., L. K. Lawrie, F. C. Winkelmann, W. F. Buhl, Y. J. Huang, C. O. Pedersen, R. K. Strand, R. J. Liesen, D. E. Fisher, M. J. Witte, et al. (2001). Energyplus: creating a newgeneration building energy simulation program. Energy and Buildings 33(4), 319-331.

Pacific Northwest National Laboratory, Richland, WA (2011). Demand Dispatch Intelligent Demand for a More Efficient Grid.

Energy Information Administration (2017). Annual energy outlook 2015.

Pacific Northwest National Laboratory (2017). Impacts of commercial building controls on energy savings and peak load reduction.

Hao, H., C. D. Corbin, K. Kalsi, and R. G. Pratt (2017a). Transactive control of commercial buildings for demand response. IEEE Transactions on Power Systems 32, 298-304.

Hao, H., C. D. Corbin, K. Kalsi, and R. G. Pratt (2017b). Transactive control of commercial buildings for demand response. IEEE Transactions on Power Systems 32(1), 774-783.

Hao, H., D. Wu, J. Lian, and T. Yang (2018). Optimal coordination of building loads and energy storage for power grid and end user services. IEEE Transactions on Smart Grid 9(5), 4335-4345.

Huang, H., L. Chen, M. Mohammadzaheri, E. Hu, and M. Chen (2013). Multi-zone temperature prediction in a commercial building using artificial neural network model. In Control and Automation (ICCA), 2013 10th IEEE International Conference on, pp. 1896-1901. IEEE.

Huang, S., A. C. L. Malara, W. Zuo, and M. D. Sohn (2018). A bayesian network model for the optimization of a chiller plant's condenser water set point. Journal of Building Performance Simulation 11(1), 36-47.

Huang, S., W. Zuo, and M. D. Sohn (2016). Amelioration of the cooling load based chiller sequencing control. Applied Energy 168, 204-215.

Inard, C., H. Bouia, and P. Dalicieux (1996). Prediction of air temperature distribution in buildings with a zonal model. Energy and buildings 24(2), $125-132$.
Jones, E., T. Oliphant, P. Peterson, et al. (2001). SciPy: Open source scientific tools for Python. [Online; accessed January 31, 2019].

Lin, Y., P. Barooah, S. Meyn, and T. Middelkoop (2015). Experimental evaluation of frequency regulation from commercial building hvac systems. IEEE Transactions on Smart Grid 6(2), 776-783.

Lin, Y., T. Middelkoop, and P. Barooah (2012). Issues in identification of control-oriented thermal models of zones in multi-zone buildings. In Decision and Control (CDC), 2012 IEEE 51st Annual Conference on, pp. 6932-6937. IEEE.

Lu, T. and M. Viljanen (2009). Prediction of indoor temperature and relative humidity using neural network models: model comparison. Neural Computing and Applications 18(4), 345-357.

Mustafaraj, G., G. Lowry, and J. Chen (2011). Prediction of room temperature and relative humidity by autoregressive linear and nonlinear neural network models for an open office. Energy and Buildings 43(6), 1452-1460.

Omer, A. M. (2009). Energy use and environmental impacts: A general review. Journal of Renewable and Sustainable Energy 1(053101).

Ruano, A. E., E. M. Crispim, E. Z. Conceiçao, and M. M. J. Lúcio (2006). Prediction of building's temperature using neural networks models. Energy and Buildings 38(6), 682-694.

Taylor, Z. T., K. Gowri, and S. Katipamula (2008). Gridlab-d technical support document: Residential end-use module version 1.0. PNNL-17694, Pacific Northwest National Laboratory, Richland, WA.

Vivian, J., A. Zarrella, G. Emmi, and M. D. Carli (2017). An evaluation of the suitability of lumpedcapacitance models in calculating energy needs and thermal behaviour of buildings. Energy and Buildings 150, 447-465.

Whittle, P. (1963). Prediction and regulation by linear least-square methods. English Universities Press London. 